\title{
ESTUDIO DE LOS ENFOQUES DE APRENDIZAJE EN ESTUDIANTES UNIVERSITARIOS DE CIENCIAS SOCIALES Y CIENCIAS DE LA SALUD DE LA UNIVERSIDAD DE SALAMANCA. UNA VISIÓN MULTIVARIANTE A TRAVÉS DE MANOVA-BIPLOT
}

\author{
Zaira J. Zárate-Santana \\ Departamento de Estadística-Universidad de Salamanca \\ Facultad de Medicina, Campus Miguel de Unamuno. Salamanca \\ zaira_zarate@usal.es \\ Ana B. Sánchez-García \\ Grupo de Investigación IGA \\ Departamento de Estadística-Universidad de Salamanca \\ Facultad de Medicina, Campus Miguel de Unamuno. Salamanca \\ carpatino@usal.es \\ Maria-Carmen Patino-Alonso \\ Grupo de Investigación IC \\ Departamento de Didáctica, Organización y Métodos de Investigación \\ Universidad de Salamanca \\ asg@usal.es
}

Recepción Artículo: 14 Febrero 2020

Admisión Evaluación: 4 marzo 2020

Informe Evaluador 1: 18 marzo 2020

Informe Evaluador 2: 20 Marzo 2020

Aprobación Publicación: 20 abril 2020

\section{RESUMEN}

Numerosos estudios han contribuido a impulsar la investigación sobre enfoques de aprendizaje en el ámbito universitario. El enfoque adoptado por los estudiantes frente a la realización de la tarea puede ser superficial 0 profundo y tiende a cambiar en función de diferentes variables contextuales. El objetivo principal de este trabajo fue estudiar el tipo de enfoque de aprendizaje que presentan los estudiantes de las áreas de Ciencias Sociales y Jurídicas y Ciencias de la Salud de la Universidad de Salamanca. La muestra objeto de estudio estuvo compuesta por 518 alumnos del área de Ciencias Sociales y Jurídicas y 300 alumnos de Ciencias de la Salud. Se utilizó el cuestionario R-SPQ-2F y se midieron las diferencias entre los enfoques de aprendizaje y las áreas de conocimiento estudiadas a través de la prueba $U$ de Mann-Whitney para muestras independientes. Para visualizar las relaciones entre los enfoques de aprendizaje y las áreas de conocimiento, se llevó a cabo el análisis multivariante Manova-Biplot. Los hallazgos obtenidos muestran que el enfoque profundo y el enfoque superficial son prácticamente independientes. El enfoque profundo predomina en el área de ciencias de la salud y se constatan diferencias significativas en este enfoque entre los estudiantes de Ciencias de la Salud y los estudiantes de Ciencias Sociales y Jurídicas.

Palabras clave: enfoques de aprendizaje; estudiantes universitarios; Ciencias de las Salud; Ciencias Jurídicas 


\title{
ESTUDIO DE LOS ENFOQUES DE APRENDIZAJE EN ESTUDIANTES UNIVERSITARIOS DE CIENCIAS SOCIALES Y CIENCIAS DE LA SALUD DE LA UNIVERSIDAD DE SALAMANCA. UNA VISIÓN MULTIVARIANTE A TRAVÉS DE MANOVA-BIPLOT
}

\begin{abstract}
Study of learning approaches in university students of social sciences and health sciences at the university of Salamanca. A multivariate vision through MANOVA-BIPLOT. Several studies have contributed to promoting research on learning approaches at the university level. The approach taken by the students can be surface or deep and can change depending on different contextual variables. The main goal of this work was to study the type of learning approach presented by students from the areas of Social and Legal Sciences and Health Sciences at the University of Salamanca. The samples under study were 518 students from the area of Social and Legal Sciences and 300 students from Health Sciences. The R-SPQ-2F questionnaire waS analyzed and the differences between the learning approaches and the areas of knowledge studied were measured using the Mann-Whitney $U$ test for independent samples. To visualize the relationships between learning approaches and knowledge areas, the Manova-Biplot multivariate analysis was carried out. The findings found that the deep approach and superficial approach are practically independent. The deep approach predominates in the area of Health Sciences and there are significant differences in this approach between students from Health Sciences and students from Social and Legal Sciences.
\end{abstract}

Keywords: learning approaches; college students; health sciences; social and legal sciences

\section{INTRODUCCIÓN}

Desde la década de los setenta han surgido diferentes estudios que han profundizado en el análisis de los enfoques de aprendizaje, lo cual ha sido un foco de atención entre los investigadores (Beyazta \& Senemo lu, 2015; Bernal García et al., 2019; Biggs, 1988, 1991; Biggs et al., 2001; González Geraldo et al., 2011; Hernández Pina et al., 2010; Kember et al., 2004; Marton \& Säljö, 1976). Los enfoques de aprendizaje surgen por primera vez en la teoría Student Approaches to Learning (SAL) de Marton \& Säljö, (1976) quienes propusieron la idea de enfoques: profundo y superficial, los cuales se refieren a diferentes intenciones de aprendizaje individuales con respecto a un tema (Hernández Pina, García and Maquilón, 2005).

El enfoque profundo, se caracteriza por la preocupación del estudiante para comprender el contenido de la tarea. El alumnado que adopta este enfoque intenta relacionar los contenidos con contextos personales significativos o con conocimientos previos y encuentra el aprendizaje emocionalmente satisfactorio (Biggs et al., 2001; Freiberg Hoffmann \& Fernández Liporace, 2016). Los resultados previos apuntan a que es característico de estudiantes creativos (Duff \& McKinstry, 2007; Warburton, 2003), que presentan habilidades de expresión, claridad de ideas, (Chin \& Brown, 2000) y motivación intrínseca (Bigss, 1988).

Por otra parte, Ios estudiantes que adoptan un enfoque superficial realizan las tareas con el mínimo esfuerzo, transmiten la idea de que el trabajo parece haberse realizado correctamente cuando no lo ha hecho, memorizan para dar la impresión de que se ha producido la comprensión (Freiberg Hoffmann \& Fernández Liporace, 2016), presentan motivación extrínseca y tienden a cumplir con los requisitos mínimos de estudio (Monroy \& Hernández Pina, 2014). Además, tienen miedo a fallar (Duff \& McKinstry, 2007).

Biggs, (1987) describe los enfoques de aprendizaje profundo y superficial en función de la motivación 0 estrategias utilizadas para abordar una tarea determinada. Ambos, motivo (Ryan \& Deci, 2000) y estrategia, forman parte del proceso de enseñanza-aprendizaje e influyen en la elección de un enfoque u otro (Freiberg Hoffmann \& Fernández Liporace, 2016). La preferencia de enfoque se relaciona con la motivación hacia el aprendizaje y por si se orienta, o no, a la comprensión de significados y ambos no pueden coexistir a la vez (GonzálezGarcía et al., 2019). La naturaleza de la relación entre estudiante, contexto y tarea se describe mediante un enfoque de aprendizaje (Monroy \& Hernández Pina, 2014), que no es estático (Ullah, 2016) y tiende a cambiar en función de diferentes variables contextuales (Dolmans et al., 2016).

Biggs, (1987, 1991); Biggs et al., (2001) y Kember et al. (2004); definen los enfoques de aprendizaje a través de un modelo de tres fases (3P): Presagio, Proceso y Producto. Donde presagio se refiere a los aspectos contextuales, el proceso a la dinámica de enseñanza-aprendizaje y el producto al resultado académico. De acuerdo a este modelo, existen cuatro componentes principales: dos de presagio, los alumnos y el contexto de apren- 
dizaje (Rosário et al., 2014). Este modelo, explica la enseñanza como un proceso interactivo y equilibrado donde todos sus elementos se interrelacionan (López Aguado \& López Alfonso, 2013), y en el que a su vez intervienen variables estratégicas, emocionales y contextuales. Las diversas corrientes y teorías de aprendizaje empírico nos permiten conocer las variables que impactan en el proceso del aprendizaje (Schunk, 2012) por lo que el estudio de las variables que expliquen las diferencias en la adopción de enfoques de aprendizaje es considerado como relevante. Estudios como los llevados a cabo por Shah, (2016) y Montealegre (2014), sitúan sus análisis a este respecto en el campo de la salud, sin embargo, no se han encontrado antecedentes que hayan indagado desde una aproximación metodológica multivariante sobre las diferencias encontradas entre las áreas de Ciencias de la Salud y Ciencias Sociales y Jurídicas y si existe, por tanto, un patrón de adopción del enfoque de aprendizaje diferencial entre ambas áreas. Por lo tanto, el principal objetivo de este trabajo fue conocer qué tipo de enfoque de aprendizaje adopta prioritariamente el alumnado universitario en las áreas de Ciencias de la Salud y Ciencias Sociales y Jurídicas de la Universidad de Salamanca, así como las posibles diferencias que se establecen entre ambos grupos.

\section{MÉTODOS}

\section{Muestra}

La muestra objeto de estudio estuvo conformada por un total de 818 alumnos de Grado que cursan estudios en la Universidad de Salamanca (USAL) distribuidos en dos grupos muestrales: estudiantes del área de Ciencias Sociales y Jurídicas $(n=518)$, y estudiantes del área Ciencias de la Salud $(n=300)$. Todos con edades comprendidas entre los 18 y los 25 años con una media de edad de 21 años. En la muestra se observa un predominio de mujeres frente a hombres en ambas áreas de conocimiento. El porcentaje de mujeres seleccionadas frente a hombres ha sido de $70,8 \%$ sobre $29,2 \%$.

\section{Instrumentos}

Para recoger la información fue aplicado el Cuestionario de Enfoques de Aprendizaje R-SPQ-2F (Biggs et al., 2001), versión reducida del SPQ (Study Process Questionnaire) de (J. Biggs, 1987) adaptado al español por (Hernández-Pina, García, \& Maquilón, 2005) y validado por Justicia, Pichardo, Cano, Garcia-Berbén, \& de la Fuente, (2008). Este cuestionario está conformado por 20 ítems divididos en dos dimensiones: superficial (10 ítems) y profundo (10 ítems). La aplicación del cuestionario fue de manera virtual, mediante un enlace a la encuesta a través de la plataforma de enseñanza virtual Studium de la Universidad de Salamanca.

\section{Análisis}

Se realizó un análisis descriptivo de cada uno de los ítems del cuestionario de enfoques de aprendizaje RSPQ-2F. El análisis de las pruebas de normalidad de las variables se llevó a cabo con el test de KolmogorovSmirnof. Para medir las diferencias entre los enfoques de aprendizaje y las áreas de conocimiento estudiadas se utilizó la prueba U de Mann-Whitney para muestras independientes. Y, por último, para visualizar las relaciones entre los enfoques de aprendizaje y las áreas de conocimiento, se llevó a cabo el análisis multivariante ManovaBiplot (Gabriel, 1972, 1995). Los datos fueron analizados con la versión 25.0 de IBM SPSS Statistical Package programme y el programa estadístico Multbiplot (Vicente-Villardón, 2017).

\section{RESULTADOS}

A continuación se presenta el análisis descriptivo de cada uno de los ítems del cuestionario R-SPQ-2F para las áreas de Ciencias Sociales y Jurídicas y Ciencias de la Salud. La tabla 1 y tabla 2 muestran los estadísticos descriptivos de todos los ítems del área de Ciencias Sociales y Jurídicas y Ciencias de la Salud respectivamente. 
Tabla 1

Análisis descriptivo de los ítems del cuestionario por R-SPQ-2F dimensión - Ciencias Sociales y Jurídicas

\begin{tabular}{lcccc}
\hline & Media \pm DS & Mediana (RI) & Asímetría & Curtosis \\
\hline Enfoque Profundo & & & & \\
EP 1 & $2,70 \pm 1,027$ & $2,00(2,00-4,00)$ & 0,294 & $-0,800$ \\
EP 2 & $3,14 \pm 1,115$ & $3,00(2,00-4,00)$ & $-0,033$ & $-0,927$ \\
EP 5 & $2,82 \pm 0,967$ & $3,00(2,00-4,00)$ & 0,203 & $-0,714$ \\
EP 6 & $2,30 \pm 0,989$ & $2,00(2,00-3,00)$ & 0,472 & $-0,394$ \\
EP 9 & $3,09 \pm 1,122$ & $3,00(2,00-4,00)$ & $-0,007$ & $-0,852$ \\
EP 10 & $3,19 \pm 1,156$ & $3,00(2,00-4,00)$ & $-0,121$ & $-0,861$ \\
EP 13 & $3,98 \pm 0,941$ & $4,00(3,00-5,00)$ & $-0,979$ & 0,213 \\
EP 14 & $2,16 \pm 0,997$ & $4,00(1,00-3,00)$ & $-0,800$ & $-0,077$ \\
EP 17 & $2,29 \pm 1,042$ & $2,00(2,00-3,00)$ & 0,683 & $-0,013$ \\
EP 18 & $3,00 \pm 1,141$ & $3,00(2,00-4,00)$ & 0,004 & $-0,924$ \\
Enfoque Superficial & & & & \\
ES 3 & $2,15 \pm 1,193$ & $2,00(2,00-4,00)$ & 0,934 & $-0,008$ \\
ES 4 & $2,80 \pm 1,269$ & $3,00(2,00-4,00)$ & 0,218 & $-1,118$ \\
ES 7 & $2,52 \pm 1,262$ & $2,00(1,00-3,25)$ & 0,420 & $-0,909$ \\
ES 8 & $2,60 \pm 1,206$ & $2,00(2,00-4,00)$ & 0,327 & $-0,890$ \\
ES 11 & $2,32 \pm 1,191$ & $2,00(1,00-3,00)$ & 0,548 & $-0,721$ \\
ES 12 & $2,53 \pm 1,082$ & $2,00(2,00-3,00)$ & 0,491 & $-0,468$ \\
ES 15 & $1,62 \pm 0,857$ & $1,00(1,00-2,00)$ & 1,408 & 1,565 \\
ES 16 & $2,64 \pm 1,195$ & $2,00(2,00-3,00)$ & 0,415 & $-0,736$ \\
ES 19 & $2,20 \pm 1,116$ & $2,00(1,00-3,00)$ & 0,729 & $-0,163$ \\
ES 20 & $1,95 \pm 1,083$ & $2,00(1,00-3,00)$ & 1,082 & 0,509 \\
\hline DS De
\end{tabular}

DS: Desviación estándar; RI: Rango intercuartílico 
Tabla 2

Análisis descriptivo de los ítems del cuestionario por R-SPQ-2F dimensión - Ciencias de la Salud

\begin{tabular}{lcccc}
\hline & Media \pm DS & Mediana (RI) & Asímetría & Curtosis \\
\hline Enfoque Profundo & & & & \\
EP 1 & $2,85 \pm 1,054$ & $3,00(2,00-4,00)$ & 0,165 & $-0,862$ \\
EP 2 & $3,61 \pm 1,043$ & $4,00(3,00-4,00)$ & $-0,420$ & $-0,754$ \\
EP 5 & $3,05 \pm 0,980$ & $3,00(2,00-4,00)$ & 0,035 & $-0,745$ \\
EP 6 & $2,42 \pm 1,033$ & $2,00(2,00-3,00)$ & 0,509 & $-0,439$ \\
EP 9 & $3,20 \pm 1,143$ & $3,00(2,00-4,00)$ & $-0,135$ & $-0,834$ \\
EP 10 & $3,65 \pm 1,050$ & $4,00(3,00-4,00)$ & $-0,524$ & $-0,493$ \\
EP 13 & $4,19 \pm 0,857$ & $4,00(4,00-5,00)$ & $-0,979$ & 0,744 \\
EP 14 & $2,17 \pm 0,975$ & $2,00(1,00-3,00)$ & 0,590 & $-0,219$ \\
EP 17 & $2,56 \pm 1,196$ & $2,00(2,00-3,00)$ & 0,402 & $-0,777$ \\
EP 18 & $3,03 \pm 1,103$ & $3,00(2,00-4,00)$ & 0,069 & $-0,849$ \\
Enfoque Superficial & & & & \\
ES 3 & $2,02 \pm 1,145$ & $2,00(1,00-3,00)$ & 0,997 & 0,036 \\
ES 4 & $2,95 \pm 1,237$ & $3,00(2,00-4,00)$ & 0,014 & $-1,126$ \\
ES 7 & $2,50 \pm 1,223$ & $2,00(1,00-3,00)$ & 0,420 & $-0,812$ \\
ES 8 & $2,33 \pm 1,098$ & $2,00(1,00-3,00)$ & 0,508 & $-0,693$ \\
ES 11 & $2,08 \pm 1,033$ & $2,00(1,00-3,00)$ & 0,914 & 0,293 \\
ES 12 & $2,41 \pm 1,045$ & $2,00(2,00-3,00)$ & 0,507 & $-0,336$ \\
ES 15 & $1,52 \pm 0,871$ & $1,00(1,00-2,00)$ & 1,955 & 3,841 \\
ES 16 & $2,71 \pm 1,286$ & $3,00(2,00-4,00)$ & 0,286 & $-0,984$ \\
ES 19 & $2,34 \pm 1,167$ & $2,00(1,00-3,00)$ & 0,620 & $-0,454$ \\
ES 20 & $1,91 \pm 1,041$ & $2,00(1,00-2,00)$ & 1,053 & 0,432 \\
\hline DS: Desv
\end{tabular}

DS: Desviación estándar; RI: Rango intercuartílico

En la tabla 3 se presentan los datos relativos a los análisis descriptivos realizados para cada uno de los factores que integran las escalas de enfoques de aprendizaje en las dos áreas de estudio consideradas. Observamos como se obtienen mayores puntuaciones en el área de Ciencias de la Salud en el enfoque de aprendizaje profundo, encontrando entre ambas áreas diferencias significativas en el enfoque profundo $(p<0.001)$.

Tabla 3 Análisis descriptivo de los ítems del cuestionario por R-SPQ-2F dimensión - Ciencias de la Salud

\section{Ciencias Sociales y Ciencias de la Salud} Jurídicas

\begin{tabular}{lccccc}
\hline & Media \pm DS & $\begin{array}{c}\text { Mediana } \\
(\mathrm{RI})\end{array}$ & Media $\pm \mathrm{DS}$ & Mediana (RI) & p-valor \\
Enfoque & $28.68 \pm 6.06$ & 29.00 & $30.73 \pm 5.74$ & 31.00 & $<0.001$ \\
Profundo & & $(24.00-$ & & $(27,00-34,00)$ & \\
& & $33.00)$ & & & \\
Enfoque & $23.33 \pm 6.61$ & 23.00 & & 22.00 & 0.275 \\
Superficial & & $(19.00-$ & $22,77 \pm 6,13$ & $(18,25-$ & \\
& & $28.00)$ & & $27,00)$ & \\
\hline
\end{tabular}

DS: Desviación estándar; RI: Rango intercuartílico 


\section{ESTUDIO DE LOS ENFOQUES DE APRENDIZAJE EN ESTUDIANTES UNIVERSITARIOS DE CIENCIAS SOCIALES Y CIENCIAS DE LA SALUD DE LA UNIVERSIDAD DE SALAMANCA. UNA VISIÓN MULTIVARIANTE A TRAVÉS DE MANOVA-BIPLOT}

En la figura 1 se muestra el MANOVA-Biplot para los grupos, representados por las áreas de conocimiento estudiadas y los factores de los enfoques de aprendizaje. Como se observa en el grafico en los estudiantes del área de conocimiento de Ciencias de la Salud predomina mayoritariamente el enfoque profundo.

Se observa en la figura que el primer eje ofrece mayor discriminación entre las áreas de conocimiento de Ciencias Sociales y Jurídicas y Ciencias de la Salud y explica el 20,6\% de la variabilidad (inercia), mientras que, en conjunto con el segundo eje, se explica prácticamente el 32,46\%.

Figura 1 Manova Biplot - Enfoques de aprendizaje/Áreas de conocimiento

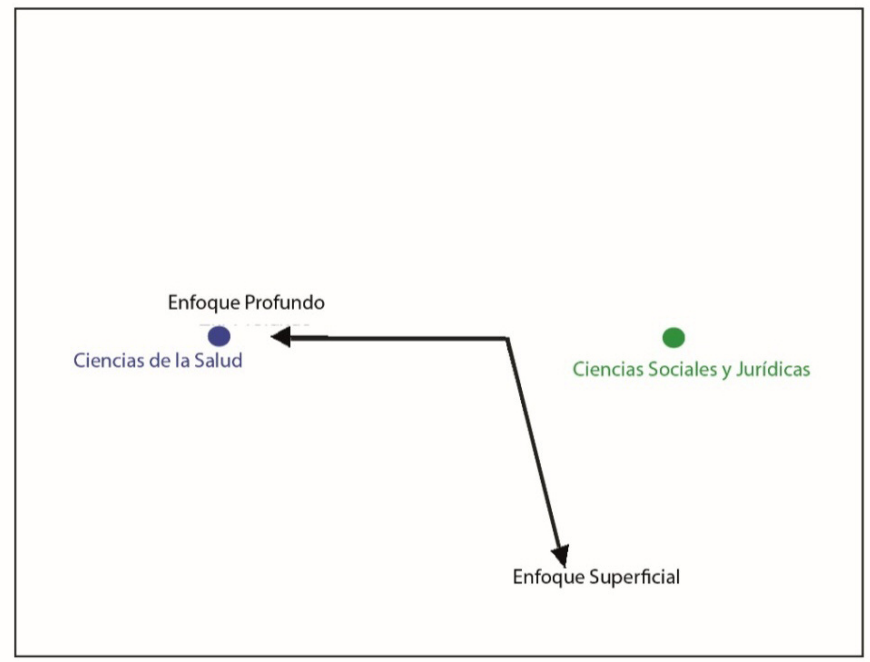

\section{DISCUSIÓN}

El objetivo principal de este trabajo fue conocer qué tipo de enfoque de aprendizaje presentan prioritariamente los estudiantes de las áreas de conocimiento de Ciencias de la Salud y Ciencias Sociales de la Universidad de Salamanca a través de un análisis descriptivo de los ítems que componen el cuestionario R-SPQ-2F y un análisis multivariante que representa las dimensiones y las áreas de conocimiento estudiadas.

Los resultados obtenidos, tal y como puede ser apreciado en la tabla 3, apuntan hacia una preferencia sobre el enfoque profundo de abordaje de la tarea frente al superficial, tanto en áreas de Ciencias de la Salud como en Ciencias Sociales y Jurídicas, luego al igual que autores como Hernández-Pina et al. (2010) se puede afirmar que el enfoque profundo es predominante en el ámbito universitario y en concreto en estas dos áreas. De acuerdo con López y López (2013) estos estudiantes en los cuales prevalece el enfoque profundo presentan ante la tarea de aprendizaje alta motivación y una comprensión significativa de la misma.

Sin embargo, cuando se realiza la comparativa entre las dos áreas en estudio consideradas se obtienen mayores puntuaciones en el área de Ciencias de la Salud en el enfoque de aprendizaje profundo, encontrando entre ambas áreas diferencias significativas en el enfoque profundo $(p<0.001)$. Resultado, que por otra parte, parece estar en la línea de otras investigaciones como Shah, (2016) y Montealegre (2014), quienes destacan que los estudiantes de Medicina, Odontología y Enfermería en su investigación también adoptan un enfoque profundo en comparación con otras áreas académicas.

Por tanto, tal y como puede ser observado en la Figura 1, con respecto al objetivo pretendido en este trabajo, los resultados apuntan hacia la existencia de un patrón diferencial entre estudiantes del área de la salud respecto 


\section{CRECIMIENTO PSICOLÓGICO Y AFRONTAMIENTO DE LA MADUREZ}

a otras áreas de conocimiento universitarias, en concreto, el área de Ciencias Sociales y Jurídicas. Si bien los resultados mostraron que hay diferencias respecto al enfoque profundo, también se detectó que en cuanto al enfoque superficial no existían diferencias estadísticamente significativas entre ambas áreas.

Finalmente, el carácter innovador de este trabajo está relacionado con la presentación por primera vez de una visión multivariante con representación gráfica de dos matrices que permiten visualizar los dos enfoques de aprendizaje conjuntamente. En este sentido, se puede afirmar tal y como se aprecia en la Figura 1, que al igual que otros autores como González-García et al. (2019) y Geraldo et al. (2011) el enfoque profundo y el enfoque superficial son prácticamente independientes.

Los resultados de esta investigación podrían utilizarse para que los profesores universitarios conozcan cuál es el enfoque de aprendizaje que predomina en sus alumnos, y así planear estrategias de enseñanza acordes a su perfil.

En general, autores como Tiwari et al. (2006) han argumentado en el contexto de enseñanza-aprendizaje universitario en Ciencias de la Salud, que la metodología de enseñanza puede influir en el enfoque de aprendizaje seleccionado por los estudiantes, por lo que más investigación es necesaria al objeto de profundizar en las diferencias metodológicas que pudieran presentarse en las áreas analizadas, y así, poder analizar si esta variable es la causa de la existencia del patrón diferencial establecido entre ambas áreas en la utilización del enfoque profundo.

Por último, con relación a las posibles limitaciones de este trabajo, debería de tomarse en consideración que la muestra de estudiantes de Ciencias de la Salud, aunque importante en número, es menor que la de estudiantes de Ciencias Sociales y Jurídicas, lo que podría tener consecuencias en términos de identificación de las preferencias de los participantes en el estudio.

\section{CONCLUSIONES}

Los resultados encontrados manifiestan que bajo la aplicación del cuestionario R-SPQ-2F, los estudiantes de la Universidad de Salamanca del área de la salud adoptan un enfoque de aprendizaje profundo encontrando diferencias significativas con los estudiantes de Ciencias Sociales y Jurídicas.

\section{REFERENCIAS BIBLIOGRÁFICAS}

Beyazta, D.I, \& Senemo lu, N. (2015). Learning Approaches of Successful Students and Factors Affecting Their Learning Approaches. Egitim ve Bilim, 40(179), 193-216. https://doi.org/10.15390/EB.2015.4214

Biggs, J. B. (1987). Study process questionnaire manual. Australian Council for Educational Research.

Biggs, J. B. (1988). Assessing student approaches to learning. Australian Psychologist, 23(2), 197-206. https://doi.org/10.1080/00050068808255604

Biggs, J. B. (1991). Approaches to learning in secondary and tertiary students in Hong Kong. Educational Research Journal, 6, 27-39.

Biggs, J., Kember, D., \& Leung, D. Y. P. (2001). The revised two-factor Study Process Questionnaire: R-SPQ-2F. British Journal of Educational Psychology, 71(1), 133-149. https://doi.org/10.1348/000709901158433

Chin, C., \& Brown, D. E. (2000). Learning in Science: A Comparison of Deep and Surface Approaches. Journal of Research in Science Teaching, 37(2), 109-138. https://doi.org/10.1002/(SICI)10982736(200002)37:2<109::AID-TEA3>3.0.C0;2-7

Dolmans, D. H. J. M., Loyens, S. M. M., Marcq, H., \& Gijbels, D. (2016). Deep and surface learning in problembased learning: a review of the literature. Advances in Health Sciences Education, 21(5), 1087-1112. https://doi.org/10.1007/s10459-015-9645-6

Duff, A., \& McKinstry, S. (2007). Students' Approaches to Learning. Issues in Accounting Education, 22(2), 183214. https://doi.org/10.2308/iace.2007.22.2.183 


\section{ESTUDIO DE LOS ENFOQUES DE APRENDIZAJE EN ESTUDIANTES UNIVERSITARIOS DE CIENCIAS SOCIALES Y CIENCIAS DE LA SALUD DE LA UNIVERSIDAD DE SALAMANCA. UNA VISIÓN MULTIVARIANTE A TRAVÉS DE MANOVA-BIPLOT}

Freiberg Hoffmann, A., \& Fernández Liporace, M. M. (2016). Enfoques de Aprendizaje según el R-SPQ-2F: Análisis de sus propiedades psicométricas en estudiantes universitarios de Buenos Aires. Revista Colombiana de Psicología, 25(2). https://doi.org/10.15446/rcp.v25n2.51874

Gabriel, K. R. (1972). Analysis of Meteorological Data by Means of Canonical Decomposition and Biplots. Journal of Applied Meteorology, 11(7), 1071-1077. https://doi.org/10.1175/15200450(1972)011<1071:AOMDBM>2.0.C0;2

Gabriel, K. R. (1995). MANOVA Biplots for two-way contingency tables (En Krzanow, pp. 227-268). Clarendon Press. Oxford, RU.

García, M. I. B., Lamos Duarte, A. F., Vargas Rivera, O. I., Camargo Villalba, G. E., \& Capacho, N. S. (2019). Enfoques de aprendizaje, rendimiento académico y factores relacionados en estudiantes que cursan último año de los programas de la Facultad de Ciencias de la Salud. Educación Médica, 20, 10-17. https://doi.org/10.1016/j.edumed.2017.11.008

Geraldo, J. L., del Rincón, B., y del Rincón, D. (2011). Estructura latente y consistencia interna del R-SPQ-2F: Reinterpretando los enfoques de aprendizaje en el EEES. Revista de Investigación Educativa, 29(2), 277-293.

González-García, N., Sánchez-García, A. B., Nieto-Librero, A. B., \& Galindo-Villardón, M. P. (2019). Attitude and Learning Approaches in the Study of General Didactics. A Multivariate Analysis. Revista de Psicodidáctica (English Ed.), 24(2), 154-162. https://doi.org/10.1016/j.psicoe.2019.03.001

González-Geraldo, J.L., del Rincón Igea, D.A. (2011). Estructura latente y consistencia interna del R-SPQ-2F: reinterpretando los enfoques de aprendizaje en el EEES. [Latent structure and internal consistency of the RSPQ-2F: Reinterpreting the approaches to learning within the EHEA]. Revista de Investigación Educativa, 29(2), 277-293

Hernández Pina, F., Rodríguez Nieto, M. C., Ruiz Lara, E., \& Esquivel Cruz, J. E. (2010). Enfoques de aprendizaje en alumnos universitarios de la titulación de ciencias de la actividad física y del deporte de España y México [Learning approaches in university students of physical activity and sport sciences in Spain and Mexico]. Revista Iberoamericana de Educación, 53(7), 1-11

Justicia, F., Pichardo, M. C., Cano, F., Berbén, A. B. G., \& De la Fuente, J. (2008). The Revised Two-Factor Study Process Questionnaire (R-SPQ-2F): Exploratory and confirmatory factor analyses at item level. European Journal of Psychology of Education, 23(3), 355-372. https://doi.org/10.1007/BF03173004

Kember, D., Biggs, J., \& Leung, D. Y. P. (2004). Examining the multidimensionality of approaches to learning through the development of a revised version of the Learning Process Questionnaire. British Journal of Educational Psychology, 74(2), 261-279. https://doi.org/10.1348/000709904773839879

López Aguado, M., \& López Alfonso, A. I. (2013). Los enfoques de aprendizaje. Revisión conceptual y de investigación. Revista Colombiana de Educación, 1(64), 131-153. https://doi.org/10.17227/01203916.64rce131.153

Marton, F., \& Säljö, R. (1976). On qualitative differences in learning: I-Outcome and process. British Journal of Educational Psychology, 46(1), 4-11. https://doi.org/10.1111/j.2044-8279.1976.tb02980.x

Monroy, F., \& Hernández Pina, F. (2014). Factores que influyen en los enfoques de aprendizaje universitario. Una revisión sistemática. Educación XX1, 17(2). https://doi.org/10.5944/educxx1.17.2.11481

Montealegre, G., Núñez, M. L., \& Salgado, D. (2014). Enfoques de aprendizajes y variables de orden sociocultural en estudiantes de una institución de educación superior en Colombia. Acta Médica Colombiana, 39(4), 368377.

Rosário, P., Núñez, J. C., Vallejo, G., Paiva, 0., Valle, A., Fuentes, S., \& Pinto, R. (2014). Are teachers' approaches to teaching responsive to individual student variation? A two-level structural equation modeling. European Journal of Psychology of Education, 29(4), 577-601. https://doi.org/10.1007/s10212-014-0214-9

Tiwari, A., Chan, S., Wong, E., Wong, D., Chui, C., Wong, A., y Patil, N. (2006). The effect of problem-based learning on students approaches to learning in the context of clinical nursing education. Nurse Education Today, 26, 430-438. 
Shah, D. K., Lochan Yadav, R., Sharma, D., Kumar Yadav, P., Islam, N., Khatri Sapkota, N., \& Kumar Jha, R. (2016). Learning approach among health sciences students in a medical college in Nepal: a cross-sectional study. Advances in Medical Education and Practice, 137. https://doi.org/10.2147/AMEP.S100968

Schunk, D. H. (2012). Teorías del aprendizaje: una perspectiva educativa [Theories of learning: an educational perspective] (6th ed.). Pearson.

Ullah, R. (2016). Learning environment, approaches to learning and learning preferences: medical students versus general education students. Journal of the Pakistan Medical Association, 66(5), 541-544.

Vicente Villardón, J.L. (2017). MULTBIPLOT: A package for Multivariate Analysis using Biplots. Departamento de Estadística. Universidad de Salamanca. 
